\title{
SOME IMPLICATIONS OF THE 2-FOLD BAILEY LEMMA
}

\author{
ALEXANDER E PATKOWSKI
}

\begin{abstract}
The 2-fold Bailey lemma is a special case of the $s$-fold Bailey lemma introduced by Andrews in 2000. We examine this special case and its applications to partitions and recently discovered $q$-series identities. Our work provides a general comparison of the utility of the 2-fold Bailey lemma and the more widely applied 1-fold Bailey lemma. We also offer a discussion of the $\operatorname{spt}_{M}(n)$ function and related identities.
\end{abstract}

Keywords: partitions; $q$-series; Bailey's lemma

2010 Mathematics Subject Classification: Primary 11P81; Secondary 11P83

\section{INTRODUCTION}

The Symmetric Bilateral Bailey transform [4], states that if

$$
B_{n}=\sum_{j=-n}^{n} A_{j} u_{n-j} v_{n+j}
$$

and

$$
\gamma_{n}=\sum_{j=|n|}^{\infty} \delta_{j} u_{j-n} v_{n+j}
$$

then

$$
\sum_{n=-\infty}^{\infty} A_{n} \gamma_{n}=\sum_{n=0}^{\infty} B_{n} \delta_{n}
$$

Here we say that $\left(A_{n}, B_{n}\right)$ is a Bailey pair (in the symmetric sense), and $\left(\gamma_{n}, \delta_{n}\right)$ is a conjugate Bailey pair. If we break symmetry, we say a pair $\left(\alpha_{n}, \beta_{n}\right)$ is a Bailey pair if

$$
\beta_{n}(a)=\sum_{j=0}^{n} \alpha_{j} u_{n-j} v_{n+j}
$$

Bailey [6] chose $v_{n}=(a q)_{n}^{-1}, u_{n}=(q)_{n}^{-1}$, (where we use standard notation [8] for $q$-shifted factorials) giving the conjugate pair $\left(\gamma_{n}, \delta_{n}\right)$

$$
\delta_{n}=\left(\rho_{1}\right)_{n}\left(\rho_{2}\right)_{n}\left(a q / \rho_{1} \rho_{2}\right)^{n},
$$

Date: 


$$
\gamma_{n}=\frac{\left(a q / \rho_{1}\right)_{\infty}\left(a q / \rho_{2}\right)_{\infty}}{(a q)_{\infty}\left(a q / \rho_{1} \rho_{2}\right)_{\infty}} \frac{\left(\rho_{1}\right)_{n}\left(\rho_{2}\right)_{n}\left(a q / \rho_{1} \rho_{2}\right)^{n}}{\left(a q / \rho_{1}\right)_{n}\left(a q / \rho_{2}\right)_{n}}
$$

In [3] Andrews considered an $s$-fold extension of the Bailey lemma, and gave the definition of the $s$-fold Bailey pair $\left(A_{n_{1}, n_{2}, \ldots, n_{s}}, B_{n_{1}, n_{2}, \ldots, n_{s}}\right)$ relative to $\left(a_{1}, a_{2}, \ldots, a_{s}\right)$,

$$
B_{n_{1}, \ldots, n_{s}}=\sum_{r_{1}=-n_{1}}^{n_{1}} \cdots \sum_{r_{s}=-n_{s}}^{n_{s}} \frac{A_{r_{1}, \ldots, r_{s}}}{\left(a_{1} q\right)_{n_{1}+r_{1}}(q)_{n_{1}-r_{1}} \ldots\left(a_{s} q\right)_{n_{s}+r_{s}}(q)_{n_{s}-r_{s}}} .
$$

Here we focus on the $s=2$ case, in which case Andrews' generalisation of (1.4) is given by

$$
B_{n_{1}, n_{2}}=\sum_{r_{1}=-n_{1}}^{n_{1}} \sum_{r_{2}=-n_{2}}^{n_{2}} \frac{A_{r_{1}, r_{2}}}{\left(a_{1} q\right)_{n_{1}+r_{1}}(q)_{n_{1}-r_{1}}\left(a_{2} q\right)_{n_{2}+r_{2}}(q)_{n_{2}-r_{2}}} .
$$

For this symmetric 2-fold Bailey pair relative to $\left(a_{1}, a_{2}\right)$ we have the identity

$$
\sum_{n_{1}=-\infty}^{\infty} \sum_{n_{2}=-\infty}^{\infty} A_{n_{1}, n_{2}} \gamma_{n_{1}}\left(a_{1}\right) \gamma_{n_{2}}\left(a_{2}\right)=\sum_{n_{1}=0}^{\infty} \sum_{n_{2}=0}^{\infty} B_{n_{1}, n_{2}} \delta_{n_{1}}\left(a_{1}\right) \delta_{n_{2}}\left(a_{2}\right),
$$

where both $\left(\gamma_{n_{1}}\left(a_{1}\right), \delta_{n_{1}}\left(a_{1}\right)\right)$ and $\left(\gamma_{n_{2}}\left(a_{2}\right), \delta_{n_{2}}\left(a_{2}\right)\right)$ are given by (1.5)-(1.6). In [3] Andrews' main focus is the application of the conjugate pair (1.5)-(1.6) for both $\left(\gamma_{n_{1}}(1), \delta_{n_{1}}(1)\right)$ and $\left(\gamma_{n_{2}}(1), \delta_{n_{2}}(1)\right)$ in (1.7) with $\rho_{1}, \rho_{2} \rightarrow \infty$. In [10] $q$-series were discovered related to both positive definite quadratic forms and indefinite ternary quadratic forms using only the 1-fold Bailey lemma. A nice consequence of [10] is that it is clear that identities that arise naturally from inserting 2-fold Bailey pairs into the 2-fold Bailey lemma may also be obtained using Bailey pairs with the 1-fold Bailey lemma. One particularly nice example from that study is the new expansion

$$
(q)_{\infty}^{3}=\sum_{N \in \mathbb{Z}}(-1)^{N} q^{N(N-1) / 2} \sum_{\substack{n=0 \\ 2|j| \leq n}}^{\infty}(-1)^{n+j} q^{n(n+1) / 2-j(3 j-1) / 2+j N} .
$$

In fact one may summarize the simple method from [10] in the following proposition.

Proposition 1. If $\left(\gamma_{n}, \delta_{n}\right)$ is a conjugate Bailey pair relative to $a=1$, then $\left(\alpha_{N}, \beta_{N}\right)$ is a Bailey pair relative to $a=q$ where

$$
\begin{gathered}
\alpha_{N}=\frac{1-q^{2 N+1}}{1-q} \sum_{j \in \mathbb{Z}} \gamma_{j}(-1)^{N+j} q^{\left(\begin{array}{c}
j+N \\
2
\end{array}\right)+j}, \\
\beta_{N}=\frac{\delta_{N} q^{-N}}{(q)_{2 N}} .
\end{gathered}
$$


Given [10], one might wonder if there is a direct relation between a 1-fold Bailey pair given a 2-fold Bailey pair. As it turns out, there are many such relations and, in general, we have the following relationship between the 1-fold and the $(s+1)$-fold Bailey pair.

Lemma 2. Let $\left(A_{n_{1}, \ldots, n_{s}, n}, B_{n_{1}, \ldots, n_{s}, n}\right)$ form an $(s+1)$-fold Bailey pair relative to $\left(a_{n_{1}}, a_{n_{2}}, \ldots, a_{n_{s}}, a\right)$, and let $\left(\gamma^{(k)}, \delta^{(k)}\right)$ for $k=1, \ldots, s$ be $s$ conjugate Bailey pairs relative to $a_{n_{k}}$. Then $\left(A_{n}, B_{n}\right)$ defined by

$$
\begin{aligned}
A_{n} & :=\sum_{n_{1}, \ldots, n_{s} \in \mathbb{Z}} \gamma_{n_{1}}^{(1)} \cdots \gamma_{n_{s}}^{(s)} A_{n_{1}, \ldots, n_{s}, n} \\
B_{n} & :=\sum_{n_{1}, \ldots, n_{s}=0}^{\infty} \delta_{n_{1}}^{(1)} \cdots \delta_{n_{s}}^{(s)} B_{n_{1}, \ldots, n_{s}, n}
\end{aligned}
$$

forms a 1-fold Bailey pair relative to a.

Proof. We write out the $s=1$ case. We have,

$$
\begin{aligned}
& \sum_{n_{1}=0}^{\infty} \delta_{n_{1}}^{(1)}\left(a_{1}\right) B_{n_{1}, n}\left(a_{1}, a\right) \\
& =\sum_{n_{1}=0}^{\infty} \delta_{n_{1}}^{(1)}\left(a_{1}\right) \sum_{r_{1}=-n_{1}}^{n_{1}} \sum_{r_{2}=-n}^{n} \frac{1}{\left(a_{1} q\right)_{n_{1}+r_{1}}(q)_{n_{1}-r_{1}}(a q)_{n+r_{2}}(q)_{n-r_{2}}} \\
& =\sum_{r_{2}=-n}^{n} \frac{A_{r_{1}, r_{2}}}{(a q)_{n+r_{2}}(q)_{n-r_{2}}} \sum_{n_{1}=0}^{\infty} \delta_{n_{1}}^{(1)}\left(a_{1}\right) \sum_{r_{1}=-n_{1}}^{n_{1}} \frac{A_{r_{1}, r_{2}}}{\left(a_{1} q\right)_{n_{1}+r_{1}}(q)_{n_{1}-r_{1}}} \\
& =\sum_{r_{2}=-n}^{n} \frac{1}{(a q)_{n+r_{2}}(q)_{n-r_{2}}} \sum_{r_{1}=-\infty}^{\infty} A_{r_{1}, r_{2}} \sum_{n_{1}=\left|r_{1}\right|}^{\infty} \frac{\delta_{n_{1}}^{(1)}\left(a_{1}\right)}{\left(a_{1} q\right)_{n_{1}+r_{1}}(q)_{n_{1}-r_{1}}} \\
& =\sum_{r_{2}=-n}^{n} \frac{1}{(a q)_{n+r_{2}}(q)_{n-r_{2}}} \sum_{r_{1}=-\infty}^{\infty} A_{r_{1}, r_{2}} \gamma_{r_{1}}^{(1)}\left(a_{1}\right) \\
& =\sum_{r_{2}=-n}^{n} \frac{A_{r_{2}}(a)}{(a q)_{n+r_{2}}(q)_{n-r_{2}}}=B_{n}(a) .
\end{aligned}
$$

Since these steps can be repeated separately for each $k$, the more general case follows.

Lemma 2 appears to be new, and allows one to prove all of Andrews' pentagonal number theorem identities in [3] using only the 1-fold Bailey lemma, by choosing $\gamma_{n_{k}}^{(k)}=q^{n_{k}^{2}} /(q)_{\infty}$, and $\delta_{n_{k}}^{(k)}=q^{n_{k}^{2}}$, for every $k$ between 1 and $s$. 
As an application of Lemma 2 we will show that Slater's well known Bailey pair (A5), see [13, p. 463], follows from the "diagonal" 2-fold Bailey pair [3, Eqs. (4.1) and (4.4)]

$$
\begin{aligned}
& A_{n_{1}, n_{2}}=(-1)^{n_{1}+n_{2}} q^{\left({ }^{n_{1}+n_{2}}\right)} \\
& B_{n_{1}, n_{2}}= \begin{cases}\frac{1}{(q)_{2 n_{1}}} & \text { if } n_{1}=n_{2} \\
0 & \text { otherwise }\end{cases}
\end{aligned}
$$

relative to 1 . First we obtain Slater's $B_{n}$ :

$$
\begin{aligned}
B_{n} & =\sum_{k=0}^{\infty} B_{k, n} q^{k^{2}} \\
& =\sum_{k=0}^{\infty} \chi(k=n) \frac{q^{k^{2}}}{(q)_{2 n}} \\
& =\frac{q^{n^{2}}}{(q)_{2 n}} .
\end{aligned}
$$

For $A_{n}$ we have to work a little bit harder:

$$
\begin{aligned}
A_{n} & =\frac{1}{(q ; q)_{\infty}} \sum_{k=-\infty}^{\infty} A_{k, n} q^{k^{2}} \\
& =\frac{(-1)^{n} q^{n(n-1) / 2}}{(q ; q)_{\infty}} \sum_{k=-\infty}^{\infty}(-1)^{k} q^{k(3 k-1) / 2+n j} .
\end{aligned}
$$

By the Jacobi triple product identity [8, Eq. (1.6.1)]

$$
\sum_{j=-\infty}^{\infty}(-a)^{j} q^{\left(\begin{array}{l}
j \\
2
\end{array}\right)}=(q)_{\infty}(a)_{\infty}(q / a)_{\infty}
$$

this yields

$$
A_{n}=\frac{(-1)^{n} q^{n(n-1) / 2}\left(q^{3}, q^{3}\right)_{\infty}\left(q^{n+1} ; q^{3}\right)_{\infty}\left(q^{2-n} ; q^{3}\right)_{\infty}}{(q ; q)_{\infty}} .
$$

considering the three congruence classes of $n$ modulo 3 this finally simplifies to

$$
\alpha_{n}= \begin{cases}1 & \text { if } n=0 \\ q^{3 k^{2}+k}+q^{3 k^{2}-k} & \text { if } n=3 k \\ -q^{3 k^{2} \pm k} & \text { if } n=3 k \pm 1 .\end{cases}
$$


Now given our computations, we can exploit the uniqueness of Bailey pairs to obtain a 2-fold Bailey pair from Slater's $A(3)$ Bailey pair. We show this by first observing that the $\alpha_{n}$ corresponding to Slaters $[13, \mathrm{~A}(3)]$ is the same as $\alpha_{n}$ in (1.16) but with $q$ replaced by $q^{2}$,

$$
\alpha_{n}= \begin{cases}1 & \text { if } n=0 \\ q^{6 k^{2}+2 k}+q^{6 k^{2}-2 k} & \text { if } n=3 k \\ -q^{6 k^{2} \pm 2 k} & \text { if } n=3 k \pm 1 .\end{cases}
$$

That is, suppose

$$
B_{n}=\frac{q^{n}}{(q)_{2 n}}
$$

and in Lemma 2, set $s=1$, and $\gamma_{n_{1}}^{(1)}=q^{n_{1}^{2}} /(q)_{\infty}$, and $\delta_{n_{1}}^{(1)}=q^{n_{1}^{2}}$. The uniqueness of Bailey pairs with (1.17) and (1.15) tells us we must have

$$
\begin{aligned}
A_{n} & =\frac{(-1)^{n} q^{n(n-1)}}{\left(q^{2} ; q^{2}\right)_{\infty}} \sum_{j \in \mathbb{Z}}(-1)^{j} q^{j(3 j-1)+2 n j} \\
& =\frac{1}{(q)_{\infty}} \sum_{r=-\infty}^{\infty} q^{r^{2}} A_{r, n}
\end{aligned}
$$

and

$$
\begin{aligned}
B_{n} & =\frac{q^{n}}{(q)_{2 n}} \\
& =\sum_{r=0}^{\infty} q^{r^{2}} B_{r, n} .
\end{aligned}
$$

Therefore, the $A_{r, n}$ and $B_{r, n}$ are forced once $\left(\gamma_{n_{1}}^{(1)}, \delta_{n_{1}}^{(1)}\right)$ is chosen, and we have proven that we must have the symmetric 2 -fold Bailey pair

$$
\begin{gathered}
A_{n_{1}, n_{2}}=\frac{(-1)^{n_{1}+n_{2}}}{(-q)_{\infty}} q^{4\left(\begin{array}{c}
n_{1} \\
2
\end{array}\right)+n_{1}+2\left(\begin{array}{c}
n_{2} \\
2
\end{array}\right)+2 n_{1} n_{2}}, \\
B_{n_{1}, n_{2}}= \begin{cases}0, & \text { if } n_{2} \neq n_{1}, \\
\frac{q^{n_{1}-n_{1}^{2}}}{(q)_{2 n_{1}}}, & \text { if } n_{1}=n_{2} .\end{cases}
\end{gathered}
$$


This pair gives us the identity due to L.J. Rogers [12, pg. 332, Eq. (13)] (from the $\rho_{1}, \rho_{2} \rightarrow \infty$ case of $\left.(1.5)-(1.6)\right)$

$$
\begin{aligned}
& \sum_{n=0}^{\infty} \frac{q^{n^{2}+n}}{(q)_{2 n}}=\frac{1}{(q)_{\infty}\left(q^{2} ; q^{2}\right)_{\infty}} \sum_{n \in \mathbb{Z}}(-1)^{n} q^{n(2 n-1)} \sum_{j \in \mathbb{Z}}(-1)^{j} q^{j(3 j-1)+2 n j} \\
& =\frac{1}{\left(q^{3}, q^{4}, q^{5}, q^{6}, q^{7} ; q^{10}\right)_{\infty}\left(q^{2}, q^{18} ; q^{20}\right)_{\infty}} .
\end{aligned}
$$

It is important to note that the literature has a large volume of 1-fold Bailey pairs, and so our argument in obtaining (1.18)-(1.19) is a more natural and potent strategy in obtaining further 2-dimensional identities.

Andrews used (1.13) to obtain a nice two-dimensional pentagonal number theorem identity [3, Theorem 2]. For more 2-fold and 3-fold Bailey pairs see Berkovich [7]. Tactically speaking, the pair (1.14)-(1.15) presently has more utility, as there are more known conjugate Bailey pairs for the 1-fold Bailey lemma (e.g. [4]). However, (1.13) would appear to encompass a larger pool of identities overall, as (1.14)-(1.15) is obtained from the limiting case $\rho_{1}, \rho_{2} \rightarrow \infty$ of (1.5)-(1.6) with (1.13).

\section{The $\operatorname{spt}(n)$ FUnCTION OF ANDREWS}

In [11], we encountered the double sum

$$
\sum_{n_{1}, n_{2}=1}^{\infty} \frac{q^{n_{1}+n_{2}}}{\left(1-q^{n_{1}}\right)^{2}\left(q^{n_{1}+1}\right)_{\infty}\left(1-q^{n_{2}}\right)^{2} \ldots\left(1-q^{n_{1}+n_{2}}\right)},
$$

and asked if the sum over $n_{2}$ had any origin from the 1-fold Bailey lemma. The proof relied on a 2-fold Bailey pair from [9]. It was also suggested there was a new generalized form of Andrews' relation [5] $\operatorname{spt}(n)=n p(n)-\frac{1}{2} N_{2}(n)$. Here $\operatorname{spt}(n)$ is the total number of appearances of the smallest parts of all the partitions of $n$, $p(n)$ is the classical unrestricted partition function, and $N_{2}(n)$ is the second AtkinGarvan moment (see (2.6) and [5] for the generating function).

Lemma 3. For $n$ and $M$ non-negative integers, $\left(\alpha_{n}, \beta_{n}\right)$ forms a Bailey pair relative to $a=1$, where

$$
\alpha_{n}=\frac{(q)_{M}^{2}(-1)^{n}\left(1+q^{n}\right) q^{n(3 n-1) / 2}}{(q)_{M-n}(q)_{M+n}}
$$

for $1 \leq n \leq M, \alpha_{n}=0$ if $n>M$,

$$
\alpha_{0}=1
$$


and

$$
\beta_{n}=\frac{(q)_{M}}{(q)_{n}(q)_{n+M}}
$$

Proof. Using the inverse relation of a Bailey pair [2] (or [15, Eq. (2.4)]),

$$
\alpha_{n}=\frac{\left(1-a q^{2 n}\right)(a)_{n}(-1)^{n} q^{n(n-1) / 2}}{(1-a)(q)_{n}} \sum_{k=0}^{n}\left(q^{-n}\right)_{k}\left(a q^{n}\right)_{k} q^{k} \beta_{k}
$$

we choose our $\beta_{n}$ to be $(2.2)$, insert into (2.3), and write

$$
(-1)^{N}\left(1+q^{N}\right) q^{\left(\begin{array}{c}
N \\
2
\end{array}\right)} \sum_{j=0}^{N} \frac{\left(q^{N}\right)_{j}\left(q^{-N}\right)_{j} q^{j}}{(q)_{j}(q)_{j+M}}=\frac{(q)_{M}(-1)^{N}\left(1+q^{N}\right) q^{N(3 N-1) / 2}}{(q)_{M-N}(q)_{M+N}}=\alpha_{N}
$$

for $N>0$, and

$$
\alpha_{0}=\frac{1}{(q)_{M}} .
$$

This follows from the $q$-Chu-Vandermonde theorem [8, Eq. (II.6)] with $(a, c, n) \mapsto$ $\left(q^{N}, q^{M+1}, N\right)$, because

$$
(q)_{M} \sum_{j=0}^{N} \frac{\left(q^{N}\right)_{j}\left(q^{-N}\right)_{j} q^{j}}{(q)_{j}(q)_{j+M}}=\frac{\left(q^{M-N+1}\right)_{N} q^{N^{2}}}{\left(q^{M+1}\right)_{N}}=\frac{(q)_{M}(q)_{M} q^{N^{2}}}{(q)_{M-N}(q)_{M+N}} .
$$

Finally, to respect the convention that $\alpha_{0}=1$, we multiply through by $(q)_{M}$.

Corollary 4. We have, for each natural number $M$,

$$
\begin{gathered}
\sum_{n=1}^{\infty} \frac{q^{n}}{\left(1-q^{n}\right)^{2}\left(1-q^{n+1}\right) \cdots\left(1-q^{n+M}\right)} \\
=\frac{1}{(q)_{M}} \sum_{n=1}^{\infty} \frac{n q^{n}}{1-q^{n}}+(q)_{M} \sum_{n=1}^{M} \frac{(-1)^{n}\left(1+q^{n}\right) q^{n(3 n+1) / 2}}{(q)_{M-n}(q)_{M+n}\left(1-q^{n}\right)^{2}} .
\end{gathered}
$$

Proof. Using the conjugate pair (1.5)-(1.6), differentiating with respect to $\rho_{1}, \rho_{2}$ and then putting $\rho_{1}, \rho_{2}=1$, we obtain

$$
\sum_{n=1}^{\infty}(q ; q)_{n-1}^{2} \beta_{n} q^{n}=\alpha_{0} \sum_{n=1}^{\infty} \frac{n q^{n}}{1-q^{n}}+\sum_{n=1}^{\infty} \frac{\alpha_{n} q^{n}}{\left(1-q^{n}\right)^{2}}
$$

Applying the Bailey pair contained in Lemma 3 to (2.5) now gives the theorem.

The $q$-series on the left side of (2.4) appeared in [11], and is the generating function for $\operatorname{spt}_{M}^{*}(n)$, the total number of appearances of the smallest parts of the number of partitions of $n$ where parts greater than the smallest plus $M$ do not occur. The first sum on the right side of (2.4) may be interpreted as $\sum_{k=0}^{n} \sigma_{1}(k) p_{M}(n-k)$, where $\sigma_{1}(n)=\sum_{d \mid n} d$, and $p_{M}(n)$ is the number of partitions of $n$ into parts $\leq M$. The limiting case $M \rightarrow \infty$ is Euler's well known formula $n p(n)=\sum_{k=0}^{n} \sigma_{1}(k) p(n-k)$, 
which is also an observation used by Andrews to obtain his $\operatorname{spt}(n)$ identity [5]. By Tannery's theorem [14, pg. 292] and [5, Eq. (3.4)], it can be seen that the limit of the second sum on the right side of Corollary 4 is

$$
\frac{1}{2} \sum_{n=1}^{\infty} N_{2}(n) q^{n}=-\frac{1}{(q)_{\infty}} \sum_{n=1}^{\infty} \frac{(-1)^{n} q^{n(3 n+1) / 2}\left(1+q^{n}\right)}{\left(1-q^{n}\right)^{2}} .
$$

The second sum on the right hand side of Corollary 4 is more complicated, and is worthy of a separate study, as any information on $\operatorname{spt}_{M}^{*}(n)$ is important to better understand $\operatorname{spt}(n)$. The case $M \rightarrow \infty$ of Corollary 4 can now be seen as $\operatorname{spt}(n)=$ $n p(n)-\frac{1}{2} N_{2}(n)$. While Corollary 4 is important in its own right, it also implies the following Bailey pair.

Lemma 5. For $n$ and $M$ non-negative integers, $\left(\alpha_{M}, \beta_{M}\right)$ forms a Bailey pair relative to $a=1$, where

$$
\alpha_{M}=\frac{(-1)^{M}\left(1+q^{M}\right) q^{M(3 M+1) / 2}}{\left(1-q^{M}\right)^{2}},
$$

for $M>0$, and

$$
\begin{gathered}
\alpha_{0}=\sum_{n=1}^{\infty} \frac{n q^{n}}{1-q^{n}} \\
\beta_{M}=\frac{1}{(q)_{M}} \sum_{n=1}^{\infty} \frac{q^{n}}{\left(1-q^{n}\right)^{2}\left(1-q^{n+1}\right) \cdots\left(1-q^{n+M}\right)} .
\end{gathered}
$$

The point of this section is that no discussion of $\operatorname{spt}_{M}^{*}(n)$ (or Corollary 4) arose until studying some identities using the 2-fold Bailey lemma.

\section{REFERENCES}

[1] G. E. Andrews, The Theory of Partitions, The Encyclopedia of Mathematics and its Applications, Vol. 2, Addison-Wesley, Reading (1976).

[2] G. E. Andrews, Multiple series Rogers-Ramanujan type identities, Pacific J. Math. 114 (1984), $267-283$.

[3] G. E. Andrews, Umbral calculus, bailey chains, and pentagonal number theorems, J. Comb. Theory, Ser. A 91 (1-2): 464-475 (2000)

[4] G.E. Andrews and S.O. Warnaar, The Bailey transform and false theta functions, Ramanujan J., 14 (2007), 173-188. 
[5] G. E. Andrews, The number of smallest parts in the partitions of $n$, J. Reine Angew. Math. 624 (2008), 133-142.

[6] W. N. Bailey, Identities of the Rogers-Ramanujan type, Proc. London Math. Soc. (2), 50 (1948), 1-10.

[7] A. Berkovich, The tri-pentagonal number theorem and related identities, Int. J. Number Theory 5 (2009), 1385-1399.

[8] G. Gasper, M. Rahman, Basic hypergeometric series, Cambridge Univ. Press, Cambridge, 2004.

[9] C.M. Joshi and Y. Vyas, Bailey Type Transforms and Applications, Jñãnãbha 45, 53-80 (2015).

[10] A. E. Patkowski, On Bailey pairs and certain q-series related to quadratic and ternary quadratic forms, Colloq. Math. 122 (2011), 265-273

[11] A. E. Patkowski, An interesting q-series related to the 4-th symmetrized rank function, Discrete Mathematics, Volume 341, Issue 11, 2018, pages 2965-2968.

[12] L. J. Rogers, Second memoir on the expansion of certain infinite products, Proc. London Math. Soc. 25 (1894) 318-343.

[13] Slater, L. J. Further identities of the Rogers-Ramanujan type, Proc. Lond. Math. Soc. (2) 54 (1952), 147-167.

[14] J. Tannery, Introduction a la Th'eorie des Fonctions dune Variable, 2 ed., Tome 1, Libraire Scientifique A. Hermann, Paris, 1904.

[15] S.O. Warnaar, 50 Years of Bailey's lemma, Algebraic Combinatorics and Applications, pp. 333-347, A. Betten et al. eds., (Springer, Berlin, 2001).

1390 Bumps River Rd.

Centerville, MA 02632

USA

E-mail: alexpatk@hotmail.com, alexepatkowski@gmail.com 